\title{
SOME INEQUALITIES OF BESSEL AND MODIFIED BESSEL FUNCTIONS
}

\author{
C. M. JOSHI and S. K. BISSU
}

(Received 22 May 1989)

Communicated by W. Moran

\begin{abstract}
Two-sided inequalties for the ratio of modified Bessel functions of first kind are given, which provide sharper upper and lower bounds than had been known earlier. Wronskian type inequalities for Bessel functions are proved, and in the sequel alternative proofs of Turan-type inequalities for Bessel and modified Bessel functions are also discussed. These then lead to a two-sided inequality for Bessel fuctions. Also incorporated in the discussion is an inequality for the ratio of two Bessel functions for $0<x<1$. Verifications of these inequalities are pointed out numerically.

1980 Mathematics subject classification (Amer. Math. Soc.) (1985 Revision): primary 33 A 40; secondary 26 D 99.

Keywords and phrases: Bessel and modified Bessel functions, Turan and Wronski type inequalities.
\end{abstract}

\section{Introduction}

Inequalites for the ratio of modified Bessel functions of the first and second kind are available in the literature. For instance, the inequality for the ratio $K_{\nu}(x) / I_{\nu}(x)$ has been used by Rosenthal [6] and Ross [7] in determining the stability of fluid motion. In Section 1 we investigate inequalities for the ratio of modified Bessel functions using Soni's [10] and Nàsell's [5] inequalities. The results obtained by us improve the lower and upper bounds for $I_{\nu}(x) / I_{\nu}(y)$ of Ross [8]. In Section 2, a proof of a Turan type inequality for Bessel functions (Szász [11]) is considered. This then leads to a two-sided

The first author's research was supported by UGC grant No. F.8-13/87(SR-III).

(C) 1991 Australian Mathematical Society 0263-6115/91 \$A2.00+0.00 
inequality for the Bessel functions. The section is concluded by showing the connection between Wronskian type and Turan type inequalities. In Section 3, Turan type inequalities are proved for modified Bessel functions by considering an identity and Nàssell's inequality. Inductive families of these inequalities give ancillary inequalites for Bessel and modified Bessel functions. Finally in Section 4 we derive additional inequalities for the Bessel functions and for the ratio of two Bessel functions. The results are then verified numerically for certain values of parameters and variables involved.

\section{1. $I_{\nu}(x) / I_{\nu}(y)$ inequalities}

The modified Bessel function of first kind

$$
I_{\nu}(x)=\sum_{m=0}^{\infty} \frac{(x / 2)^{\nu+2 m}}{m ! \Gamma(\nu+m+1)},
$$

is real valued for $\nu$ real on the domain $x>0$ and it is positive for $\nu \geq-1$ on the same domain. The inequality

$$
0<I_{\nu+1}(x)<I_{\nu}(x), \quad \text { where } \nu>-\frac{1}{2} \text { and } x>0
$$

was established by Soni [10]. Jones [3] proved the stronger inequality $I_{\mu}(x)<$ $I_{\nu}(x)$, while Cochran [1] established the inequality $\partial I_{\nu}(x) / \partial \nu<0$, both for $\mu>\nu \geq 0$ and $x>0$.

Nàsell [5] easily proved a result that strengthens (1.1) for $\nu>0$, namely

$$
\left(1+\frac{\nu}{x}\right) I_{\nu+1}(x)<I_{\nu}(x) ; \quad \nu>-1 \text { and } x>0 .
$$

Násell $[5,(11)]$ bounded $I_{\nu}(x) / I_{\nu+1}(x)$ from above. But application of this to $I_{\nu}(x) / I_{\nu}(y)$ does not improve the inequality [8, (4)] of Ross. This naturally suggests investigation of inequality (1.1). Using the recurrence relation (Watson [13, (4)])

$$
z I_{\nu}^{\prime}(z)-\nu I_{\nu}(z)=z I_{\nu+1}(z)
$$

we put (1.1) in the form

$$
0<\frac{I_{\nu}^{\prime}(z)}{I_{\nu}(z)}-\frac{\nu}{z}<1 .
$$

Integrating (1.4) for $0<z \leq x$ and exponentiating we have, on checking $\lim _{x \rightarrow 0^{+}} I_{\nu}(x) / x^{\nu}$, that

$$
1<\frac{I_{\nu}(x)}{x^{\nu} 2^{\nu} \Gamma(\nu+1)}<e^{x}
$$


TABLE 1

\begin{tabular}{|r|rrr|rr|r|r|}
\hline & $\nu$ & $x$ & $y$ & \multicolumn{2}{|c|}{ Ross's inequality } & $\begin{array}{r}\text { inequality } \\
(1.5)\end{array}$ & $\begin{array}{r}\text { inequality } \\
(1.6) \\
\text { lower } \\
\text { bound }\end{array}$ \\
\hline 1 & 0.25 & 0.1 & 0.2 & 0.7608745 & 0.9293342 & 0.84102128 & 0.8102128 \\
bound & upper & bound & bound & (1) \\
3 & 1.50 & 0.5 & 1.3 & 0.1071776 & 0.5308544 & 0.2385283 & 0.1775399 \\
3 & 6.00 & 1.5 & 2.1 & 0.0728878 & 0.2419961 & 0.1328103 & 0.1156637 \\
\hline
\end{tabular}

Similarly, integration over $0<z \leq y-x$, and exponentiation yields

$$
e^{x-y}\left(\frac{x}{y}\right)^{\nu}<\frac{I_{\nu}(x)}{I_{\nu}(y)}<\left(\frac{x}{y}\right)^{\nu}
$$

where $y>x>0, \nu>-\frac{1}{2}$, which extends Ross's inequality to $-\frac{1}{2}<\nu \leq 0$.

A further extension of the inequality for $\nu$ lying in $\left(-1,-\frac{1}{2}\right]$ is possible, by the same method. Proceeding from (1.2) and (1.3), we are led similarly to the improved lower bound

$$
e^{x-y}\left(\frac{y+\nu}{x+\nu}\right)^{\nu}\left(\frac{x}{y}\right)^{\nu}<\frac{I_{\nu}(x)}{I_{\nu}(y)}
$$

where $y>x>0, \nu>-1$. See Table 1 for sample values.

The inequality

$$
\frac{K_{\nu}(x)}{K_{\nu}(y)}>\frac{I_{\nu}(x)}{I_{\nu}(y)}>e^{x-y}\left(\frac{x}{y}\right)^{\nu}, \quad y>x>0, \nu>-\frac{1}{2},
$$

is obtained in the same manner using the relation in Watson [13, (20) and(4)].

\section{Wronski and Turan inequalities for $J_{\nu}(x)$}

The Bessel functions $J_{\nu}(x)$ of order $\nu$ is defined for $\nu>-1$ by the power series

$$
J_{\nu}(x)=\sum_{k=0}^{\infty} \frac{(x / 2)^{\nu+2 k}(-1)^{k}}{k ! \Gamma(\nu+k+1)} .
$$

Szász [11] proved the inequality

$$
\Delta_{\nu}(x)=J_{\nu}^{2}(x)-J_{\nu-1}(x) J_{\nu+1}(x)>J_{\nu}^{2}(x) /(\nu+1) .
$$

Now $J_{\nu}(x)$ satisfies the recurrence relation

$$
x J_{\nu-1}(x)-\nu J_{\nu}(x)=x J_{\nu}^{\prime}(x),
$$


from which a simplified proof of (2.2) can be found. From

$$
f_{\nu}(x)=\Gamma(\nu+1)(2 / x)^{\nu} J_{\nu}(x)=f_{\nu}(-x), \quad \nu>0, x \text { real },
$$

and from (2.3) follows the difference-differential equation

$$
f_{\nu}^{\prime}(x)=\frac{2 \nu}{x}\left[f_{\nu-1}(x)-f_{\nu}(x)\right]
$$

Again let

$$
F_{\nu}(x)=f_{\nu}^{2}(x)-f_{\nu-1}(x) f_{\nu+1}(x),
$$

which like $\Delta_{\nu}(x)$ can be written as a $2 \times 2$ determinant. Expressions of the form $\phi_{a} \phi_{b}-\phi_{c} \phi_{d}$, where $a+b=c+d$, can be classified as Turan type, and those of the form $\phi_{a} \phi_{b}^{\prime}-\phi_{a}^{\prime} \phi_{b}$ as Wronskian type. Thus (2.2) and (2.6) are of Turan type.

Now (2.5) and (2.6) easily imply that

$$
\left[x^{2 \nu+2} F_{\nu}(x)\right]^{\prime}=-\frac{2 x^{2 \nu+2}}{\nu} f_{\nu}(x) f_{\nu}^{\prime}(x),
$$

and that the critical points of $x^{2 \nu+2} F_{\nu}(x)$ satisfy $x=0$ or $f_{\nu}(x)=0$ or $f_{\nu}^{\prime}(x)=0$. When $f_{\nu}(x)=0$,

$$
F_{\nu}(x)=-f_{\nu-1}(x) f_{\nu+1}(x)=\frac{4 \nu(\nu+1)}{x^{2}} f_{\nu-1}^{2}(x)>0 .
$$

When $f_{\nu}^{\prime}(x)=0,(2.5)$ implies $F_{\nu}(x)=f_{\nu}^{2}(x)>0$. Hence

$$
F_{\nu}(x)=\Gamma^{2}(\nu+1)(2 / x)^{2 \nu}\left[J_{\nu}^{2}(x)-\frac{\nu+1}{\nu} J_{\nu-1}(x) J_{\nu+1}(x)\right]>0,
$$

which implies $(2.2)$ for $x>0$.

An upper bound for $\Delta_{\nu}(x)$ also follows from one for $F_{\nu}(x)$. Obviously (2.7) can be rewritten as

$$
\left(F_{\nu}(x)+\frac{1}{\nu} f_{\nu}^{2}(x)\right)^{\prime}=-\frac{2(\nu+1)}{x} F_{\nu}(x) .
$$

From (2.2) and (2.8), $2(\nu+1) F_{\nu}(x) / x$ is positive and so

$$
F_{\nu}(x)<\frac{1}{\nu}\left(1-f_{\nu}^{2}(x)\right) .
$$

From (2.9) and (2.4), (2.5), (2.6), we have

$$
\Delta_{\nu}(x)<\frac{1}{\nu}\left(1-(2 / x)^{2 \nu} \Gamma^{2}(\nu+1) J_{\nu}^{2}(x)\right)+\frac{1}{\nu+1} J_{\nu}^{2}(x),
$$

where $\nu>0, x$ is real. 
An easy induction on (2.2) yields

$$
\begin{gathered}
J_{\nu+k}(x) J_{\nu-k}(x)-J_{\nu-k-1}(x) J_{\nu+k+1}(x) \\
>\frac{2 k+1}{\nu+k+1} J_{\nu+k}(x) J_{\nu-k}(x),
\end{gathered}
$$

where $0 \leq k<[\nu]=n, 0<\nu-[\nu]<1$.

Summation over the differences in (2.11) leads to

$$
J_{\nu}^{2}(x)-J_{\nu+n}(x) J_{\nu-n}(x)>\sum_{k=0}^{n-1} \frac{2 k+1}{\nu+k+1} J_{\nu+k}(x) J_{\nu-k}(x),
$$

where $\nu>0, x$ is real and $n \geq 1$.

Thiruvenkatachar and Najundiah [12] have proved $\Delta_{\nu}(x)>0$ by arguing that $\operatorname{sgn}\left[\left(x^{2} \Delta_{\nu}(x)\right)^{\prime}\right]=\operatorname{sgn} x$. On the other hand, Skovgaard discusses the positivity of $\Delta_{\nu}(x)$ through real zeros of $J_{\nu}(x)$. Using the recurrences for $J_{\nu}(x)$, it follows readily that

$$
\left[x^{2} \Delta_{\nu} x\right]^{\prime}=2 x J_{\nu}^{2}(x) \text {. }
$$

Lommel's result (Watson [13, (5.51)])

$$
\int_{0}^{x} t J_{\nu}^{2}(t) d t=2 \sum_{n=0}^{\infty}(\nu+2 n+1) J_{\nu+2 n+1}^{2}(x)
$$

trivially implies

$$
\Delta_{\nu}(x)>\frac{4(\nu+1)}{x^{2}} J_{\nu+1}^{2}(x), \quad \nu>-1,-\infty<x<\infty,
$$

which strengthens (2.2) for small $x$. Finally note that using [13, page 480], we can write $\Delta_{\nu}(x)$ as

$$
\Delta_{\nu}(x)=-\frac{1}{x}\left|\begin{array}{cc}
J_{\nu}(x) & x J_{\nu}^{\prime}(x) \\
\left|J_{\nu}^{\prime}(x)\right| & \left|\left(x J_{\nu}^{\prime}(x)\right)^{\prime}\right|
\end{array}\right|>0 .
$$

Further define the Wronskian

$$
G_{\nu}(x)=J_{\nu}(x) J_{\nu+1}^{\prime}(x)-J_{\nu}^{\prime}(x) J_{\nu+1}(x) .
$$

Then

$$
x^{2} G_{\nu}^{\prime}(x)=x^{2} J_{\nu}(x) J_{\nu+1}^{\prime \prime}(x)-x^{2} J_{\nu+1}(x) J_{\nu}^{\prime \prime}(x) .
$$

This can be written as

$$
\left[x G_{\nu}(x)\right]^{\prime}=\left(\frac{1+2 \nu}{x}\right) J_{\nu}(x) J_{\nu+1}(x),
$$

which implies that $G_{\nu}(x)>0$, since relative extrema of $x G_{\nu}(x)$ occur when either $J_{\nu}(x)=0$ or $J_{\nu+1}(x)=0$. If $J_{\nu}(x)=0$, then $[13,3.2,(4)]$ implies 
$G_{\nu}(x)=J_{\nu+1}^{2}(x)$; if $J_{\nu+1}(x)=0,(2.16)$ and [13, 3.2, (2)] implies $G_{\nu}(x)=$ $\frac{1}{2} J_{\nu}^{2}(x)$. Hence $G_{\nu}(x)>0$ for $\nu>0,-\infty<x<\infty$ and $x \neq 0 ; G_{\nu}(0)=$ 0 . But writing the values of $J_{\nu}^{\prime}(x)$ and $J_{\nu+1}^{\prime}(x)$ successively from (2.3) and also from [13, 3.2, (4)], (2.16) leads to the Turan forms:

$$
G_{\nu}(x)-\frac{1}{x} J_{\nu}(x) J_{\nu+1}(x)=\left|\begin{array}{cc}
J_{\nu+1}(x) & J_{\nu+2}(x) \\
J_{\nu}(x) & J_{\nu+1}(x)
\end{array}\right|
$$

and

$$
G_{\nu}(x)+\frac{1}{x} J_{\nu}(x) J_{\nu+1}(x)=\left|\begin{array}{cc}
J_{\nu}(x) & J_{\nu+1}(x) \\
J_{\nu-1}(x) & J_{\nu}(x)
\end{array}\right| .
$$

Now (2.2), (2.3), (2.17), (2.18) and (2.19) imply the inequalities

$$
\left|\begin{array}{ll}
J_{\nu-1}(x) & J_{\nu}(x) \\
J_{\nu-1}^{\prime}(x) & J_{\nu}^{\prime}(x)
\end{array}\right|>\frac{1}{x} J_{\nu-1}(x) J_{\nu}(x),
$$

and

$$
\left|\begin{array}{cc}
J_{\nu}(x) & J_{\nu+1}(x) \\
J_{\nu-1}(x) & J_{\nu}(x)
\end{array}\right|>\frac{1}{x} J_{\nu}(x) J_{\nu+1}(x),
$$

where $\nu>0,-\infty<x<\infty, x \neq 0$.

\section{Turan inequality for modified Bessel functions}

Thiruvenkatachar and Najundiah have proved

$$
0 \leq S_{\nu}(x) \leq I_{\nu}^{2}(x) /(\nu+1)
$$

where

$$
S_{\nu}(x)=I_{\nu}^{2}(x)-I_{\nu-1}(x) I_{\nu+1}(x),
$$

by comparing the coefficients in the Cauchy product $[12,(3.5)]$

$$
I_{\lambda}(x) I_{\mu}(x)=\sum_{n=0}^{\infty}\left(\begin{array}{c}
\lambda+\mu+2 n \\
n
\end{array}\right) C_{\lambda, n} C_{\mu, n}(x / 2)^{\lambda+\mu+2 n},
$$

where

$$
C_{\lambda, n}=\frac{1}{\Gamma(\lambda+n+1)} .
$$

We examine an alternate derivation of (3.1) and provide a mild extension via Nàsell's inequality (1.2). Recall that $I_{\nu}(x)$ satisfies the following recurrence relations

$$
x I_{\nu}^{\prime}(x)+\nu I_{\nu}(x)=x I_{\nu-1}(x)
$$




$$
I_{\nu-1}(x)-I_{\nu+1}(x)=\frac{2 \nu}{x} I_{\nu}(x) .
$$

Putting values of $I_{\nu-1}(x)$ and $I_{\nu+1}(x)$ in (3.2) in terms of $I_{\nu}(x)$ and $I_{\nu}^{\prime}(x)$, we have

$$
S_{\nu}(x)=\frac{I_{\nu}^{2}(x)}{x}\left(x \frac{I_{\nu}^{\prime}(x)}{I_{\nu}(x)}\right)^{\prime}
$$

Using the factorisation [13, page 498], we have

$$
I_{\nu}(x)=\left((x / 2)^{\nu} / \Gamma(\nu+1)\right) \prod_{n=1}^{\infty}\left(1+\frac{x^{2}}{j_{\nu, n}^{2}}\right),
$$

where $\pm j_{\nu, n}$ is the $n$th zero of $J_{\nu}(x) / x^{\nu}$, we have, from the logarithmic derivative, that

$$
\left(x \frac{I_{\nu}^{\prime}(x)}{I_{\nu}(x)}\right)^{\prime}=\sum_{n=1}^{\infty} \frac{4 x j_{\nu, n}^{2}}{\left(x^{2}+j_{\nu, n}^{2}\right)^{2}},
$$

and hence

$$
S_{\nu}(x)=4 I_{\nu}^{2}(x) \sum_{n=1}^{\infty} \frac{j_{\nu, n}^{2}}{\left(x^{2}+j_{\nu, n}^{2}\right)^{2}} \geq 0, \quad \nu>0,-\infty<x<\infty .
$$

From [13, 15.3,(5)], $j_{\nu, 1}>(\nu(\nu+2))^{1 / 2}$ for all $\nu \geq 0$. From tables [13, pages 748-751], $j_{0,1}>2.4, j_{1,1}>3.8, j_{2,1}>5, j_{3,1}>6, j_{4,1}>7.5$, and for $\nu \geq 4$,

Hence

$$
\frac{4}{\nu(\nu+2)}<\frac{1}{(\nu+1)}
$$

Thus

$$
\frac{4}{j_{\nu, 1}^{2}}<\frac{1}{\nu+1} \text { for all } \nu \geq 0 \text {. }
$$

$$
S_{\nu}(x) \leq I_{\nu}^{2}(x) \frac{4}{j_{\nu, 1}^{2}} \leq \frac{I_{\nu}^{2}(x)}{\nu+1}
$$

A slightly more general form of (3.9) may be written as

$$
\begin{aligned}
0 & \leq I_{\nu+k-1}(x) I_{\nu-k+1}(x)-I_{\nu-k}(x) I_{\nu+k}(x) \\
& \leq \frac{2 k-1}{\nu+k} I_{\nu+k-1}(x) I_{\nu-k+1}(x), \quad 1 \leq k<[\nu]+1,
\end{aligned}
$$

leading in particular to the summed inequality

$$
\begin{aligned}
0 & \leq I_{\nu}^{2}(x)-I_{\nu+n}(x) I_{\nu-n}(x) \\
& \leq \sum_{k=1}^{n} \frac{2 k-1}{\nu+k} I_{\nu+k-1}(x) I_{\nu-k+1}(x), \quad \nu>0,-\infty<x<\infty, n \geq 1 .
\end{aligned}
$$


In (3.9), the upper bound admits improvement when $x>1$, for from (1.2) we have

$$
\left(1+\frac{\nu}{x}\right) I_{\nu+1}(x)<I_{\nu}(x), \quad \nu>-1, x>0,
$$

or equivalently

$$
\left(1+\frac{\nu-1}{x}\right) I_{\nu}(x)<I_{\nu-1}(x), \quad \nu>0, x>0 .
$$

Combining (3.12) and (3.13), we have

$$
0<S_{\nu}(x)<\frac{1}{\nu+x} I_{\nu}^{2}(x), \quad \nu>0, x>0
$$

which admits a further extension in the form

$$
\begin{aligned}
0 & <I_{\nu+k-1}(x) I_{\nu-k+1}(x)-I_{\nu-k}(x) I_{\nu+k}(x) \\
& <\frac{2 k-1}{\nu+k+x-1} I_{\nu+k-1}(x) I_{\nu-k+1}(x), \\
& 1 \leq k<[\nu]+1, \nu>0, x>0,
\end{aligned}
$$

which yields the summed inequality

$$
\begin{aligned}
0 & <I_{\nu}^{2}(x)-I_{\nu-n}(x) I_{\nu+n}(x) \\
& <\sum_{k=1}^{n} \frac{2 k-1}{(\nu+k+x-1)} I_{\nu+k-1}(x) I_{\nu-k+1}(x), \quad \nu>0, x>0, n \geq 1 .
\end{aligned}
$$

\section{Bessel inqualities in $0<x<1$}

The inequality

$$
J_{\nu}(x)>J_{\nu+1}(x), \quad \text { for } 0<x<1, \nu>-\frac{1}{2},
$$

can be deduced from the series (Erdélyi [2, page 14], see (3))

$$
J_{\nu}(x)=\pi^{-\frac{1}{2}}(x / 2)^{\nu} \sum_{m=0}^{\infty} \frac{(-1)^{m} x^{2 m} \Gamma(m+1 / 2)}{(2 m) ! \Gamma(\nu+m+1)} .
$$

Inequality (4.1) admits further improvement in the interval $0<x<1, \nu\rangle$ 0 . We observe from (2.1) that the expression $\frac{x}{2}\left(J_{\nu}(x)\right)-J_{\nu+1}(x)$ is an alternating series of decreasing terms if $0<x<(4 \nu(\nu+2) /(\nu+1))^{1 / 2}$, and consequently the inequality

$$
J_{\nu+1}(x)<\frac{x}{2} J_{\nu}(x), \quad 0<x<\left(\frac{4 \nu(\nu+2)}{(\nu+1)}\right)^{1 / 2}, \nu>\frac{1}{7} .
$$


Hence by $J_{\nu}^{\prime}(x)=-J_{\nu+1}(x)+\nu J_{\nu}(x) / x$,

$$
0<\frac{\nu}{x}-\frac{J_{\nu}^{\prime}(x)}{J_{\nu}(x)}<\frac{x}{2}
$$

Integrating between the limits $\left(x_{1}, y\right)$, one gets

$$
\left(\frac{x_{1}}{y}\right)^{\nu}<\frac{J_{\nu}\left(x_{1}\right)}{J_{\nu}(y)}<\left(\frac{x_{1}}{y}\right)^{\nu} e \frac{y^{2}-x_{1}^{2}}{4}, \quad 0<x_{1}<y \leq 1, \nu>\frac{1}{7} .
$$

The numerical computation appended below verifies these ratios under suitable restrictions, and gives bounds of ratios not otherwise readily available.

For example

$$
.5947<\frac{J_{.75}(\cdot 5)}{J_{.75}(1)}=.6642<.7172
$$

and

$$
.7377<\frac{J_{.75}(\cdot 2)}{J_{.75}(\cdot 3)}=.7430<.7470
$$

Also

$$
.3536<\frac{J_{1.5}(\cdot 5)}{J_{1.5}(1)}<.4264
$$

and

$$
.4141<\frac{J_{1.5}(\cdot 5)}{J_{1.5}(\cdot 9)}<.4763
$$

\section{Acknowledgement}

Our sincere thanks are due to the referee for his keen interest and for suggesting a number of improvements. Also the first author records his sincere thanks to the UGC for providing financial assistance for the project.

\section{References}

[1] J. A. Cochran, 'The monotonicity of modified Bessel functions with respect to their order', J. Math. Phys. 46 (1967), 220-222.

[2] A. Erdélyi, Higher transcendental functions, Vol. II, (McGraw-Hill, New York, 1953).

[3] A. L. Jones, 'An extension of an inequality involving modified Bessel functions', J. Math. Phys. 47 (1968), 220-221.

[4] Y. L. Luke, Integrals of Bessel functions, (McGraw-Hill, New York, 1962).

[5] I. Nàsell, 'Inequalities for modified Bessel functions', Math. Comp. 28 (1975), 253-256. 
[6] D. K. Rosenthal, 'The shape and stability of a bubble at the axis of a rotating liquid', J. Fluid Mech. 12 (1962), 358-366.

[7] D. K. Ross, 'The stability of a column of liquid to torsial oscillations', Z. Angew. Math. Phys. 21 (1970), 137-140.

[8] D. K. Ross, 'Inequalities for special functions', Problem 72-15, SIAM Rev. 15 (1973), 668-670.

[9] H. Skovgaard, 'On inequalities of Turan type', Math. Scand. 2 (1954), 65-73.

[10] R. P. Soni, 'On an inequality for modified Bessel functions', J. Math. Phys. 44 (1965), 406-407.

[11] O. Szàsz, 'Inequalities concerning ultraspherical polynomials and Bessel functions', Proc. Amer. Math. Soc. 1 (1950), 256-267.

[12] V. K. Thiruvenkatachar, and T. S. Najundiah, 'Inequalities concerning Bessel functions and orthogonal polynomials', Proc. Indian Nat. Acad. Part A 33 (1951), 373-384.

[13] G. N. Watson, $A$ treatise on the theory of Bessel functions, 2nd ed., (Cambridge Univ. Press, 1944).

\section{Sukhadia University}

Udaipur 313001

India 